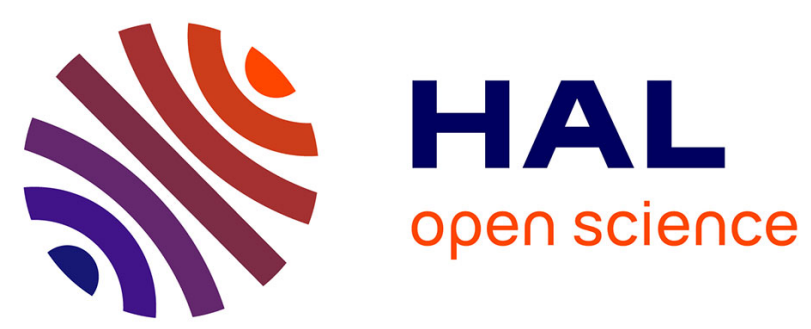

\title{
Éléments finis d'ordre deux pour l'inéquation variationnelle de Signorini
}

Zakaria Belhachmi, Faker Ben Belgacem

\section{To cite this version:}

Zakaria Belhachmi, Faker Ben Belgacem. Éléments finis d'ordre deux pour l'inéquation variationnelle de Signorini. Comptes Rendus de l'Académie des Sciences - Series I - Mathematics, 2000, 331 (9), pp.727-732. 10.1016/S0764-4442(00)01700-6 . hal-01352443

\section{HAL Id: hal-01352443 \\ https://hal.science/hal-01352443}

Submitted on 8 Aug 2016

HAL is a multi-disciplinary open access archive for the deposit and dissemination of scientific research documents, whether they are published or not. The documents may come from teaching and research institutions in France or abroad, or from public or private research centers.
L'archive ouverte pluridisciplinaire HAL, est destinée au dépôt et à la diffusion de documents scientifiques de niveau recherche, publiés ou non, émanant des établissements d'enseignement et de recherche français ou étrangers, des laboratoires publics ou privés. 


\title{
Éléments finis d'ordre deux pour l'inéquation variationnelle de Signorini
}

\author{
Zakaria BELHACHMI $^{\text {a }}$, Faker BEN BELGACEM ${ }^{\text {b }}$ \\ a Département de mathématiques, Université de Metz, Ile du Saulcy, 57045 Metz cedex 01, France \\ Courriel : belhach@poncelet.sciences.univ-metz.fr \\ ${ }^{b}$ Mathématiques pour l'industrie et la physique, UMR CNRS, UPS-INSAT-UT1 (UMR 5640), \\ Université Paul-Sabatier, 118, route de Narbonne, 31062 Toulouse cedex 04, France \\ Courriel : belgacem@mip.ups-tlse.fr
}

\begin{abstract}
Résumé. Nous présentons ici deux manières de modéliser numériquement les conditions de contact de Signorini pour des éléments finis de Lagrange quadratiques. L'analyse mathématique de leurs performances conduit à des taux de convergence optimaux énoncés dans cette Note.
\end{abstract}

\section{Finite elements of order two for Signorini's variational inequality}

\begin{abstract}
We describe here two numerical models for Signorini's contact conditions with Lagrange quadratic finite elements. The mathematical analysis for the methods leads to optimal convergence rates given in this Note.
\end{abstract}

\section{Abridged English version}

\section{Introduction}

The numerical simulation of variational inequalities arising from unilateral contact by finite element methods is source of technical difficulties both in the numerical modeling of contact conditions and in the analysis of the approximation performances. For such problems, typically represented by the Signorini model, the main question is to take into account the non-penetration in an easy way while ensuring a satisfactory accuracy of the computations (see [1,4,7]). For affine finite elements, three numerical models of unilateral contact are considered in the practice; the numerical analysis of their efficiency is given in [2]. Most often, the regularity of exact Signorini solution is better than $\mathrm{H}^{2}$ (near the contact zone) and it is worth to resort to higher order finite elements. This Note presents the two variational quadratic finite element approximations of the Signorini problem analyzed in [3], and we give optimal convergence rates obtained under reasonable assumptions on the regularity of exact solutions. In addition to the optimal results allowed by this discretizations, their numerical implementation is easy. 


\section{Signorini problem}

Let $\Omega$ be a bounded domain in $\mathbb{R}^{2}$, with Lipschitz boundary $\partial \Omega$ which is assumed to be the union of a Dirichlet portion $\Gamma_{u}$, a Neumann portion $\Gamma_{g}$ and a contact zone $\Gamma_{C}$ with a rigid obstacle. For any $f \in \mathrm{L}^{2}(\Omega)$ and $g \in \mathrm{L}^{2}\left(\Gamma_{g}\right)$, the Signorini problem consists in finding $u$ such that:

$$
\begin{array}{rlrl}
-\Delta u & =f \quad & \text { in } \Omega, \\
u=0 \quad \text { on } \Gamma_{u}, \quad \frac{\partial u}{\partial \boldsymbol{n}} & =g & & \text { on } \Gamma_{g}, \\
u \geqslant 0, \quad \frac{\partial u}{\partial \boldsymbol{n}} \geqslant 0, \quad u \frac{\partial u}{\partial \boldsymbol{n}}=0 & & \text { on } \Gamma_{C} .
\end{array}
$$

The functional framework well suited to solve problem (1) consists in working with a closed convex subset $K(\Omega)$ of the Sobolev space $\mathrm{H}^{1}(\Omega)$, defined in (2), where the Dirichlet and contact conditions are incorporated. The variational formulation of this problem yields the characterization of $u \in K(\Omega)$ as the solution of the variational inequality (3) which is well posed by Stampacchia theorem.

\section{Quadratic finite element approximations of the Signorini problem}

Let $\mathcal{T}_{h}$ be a given regular mesh of $\Omega$ made of either triangular or quadrangular elements, the first approximation of (3) is based on the standard Lagrangian finite elements, where the discrete solution is a continuous piecewise $\mathcal{P}_{2}$ (or $Q_{2}$ for quadrangles) polynomials. In the definition of the discrete closed convex cone $K_{h}(\Omega)$ the prescribed Dirichlet boundary conditions are taken into account so as the contact conditions. They are expressed by enforcing on the contact zone $\Gamma_{C}$ the nonnegativity of the approximated solution at the vertices of the elements and of the momentums on edges. The discrete variational inequality (4) set in $K_{h}(\Omega)$ is well posed by Stampacchia theorem. The numerical analysis of this approximation relies on the so-called Falk's lemma, which shows two contributions to the global bound of the error, the standard best approximation error and the consistency error due to the non-conformity of the approach. The final convergence rates are given in Theorems 1 and 2 below and are proven in [3].

For the second method the nonnegativity is enforced on the values of the discrete solution at all Lagrange degrees of freedom located in the contact zone (namely the vertices and the middle points of the edges on $\left.\Gamma_{C}\right)$. This approach seems more "natural" for implementation; it allows to enforce the condition $u_{\mid \Gamma_{C}} \geqslant 0$ rather than $\left.\frac{\partial u}{\partial \boldsymbol{n}}\right|_{\Gamma_{C}} \geqslant 0$ which is satisfied in a weak sense by the variational formulation. The corresponding discrete variational inequality (6), set in the new discrete convex cone $\widetilde{K}_{h}(\Omega)$ is also well posed. Thanks to Simpson quadrature formula it is easy to see that $\widetilde{K}_{h}(\Omega) \subset K_{h}(\Omega)$. The major consequence is that the consistency error remains unchanged while bounding the second part (the approximation error - the first term of (5)) requires more technical work. Finally, the error analysis in this case yield asymptotically the same optimal convergence rates as in the first approach and Theorems 1 and 2 are also valid for this method.

\section{Introduction}

Le développement d'outils de calcul par éléments finis pour les inéquations variationnelles modélisant des problèmes unilatéraux est source de difficultés techniques tant sur le plan de la modélisation numérique que sur le plan de l'analyse de la qualité de l'approximation. La question de savoir comment modéliser simplement les conditions de contact unilatéral (de Signorini) pour obtenir la meilleur précision possible constitue un souci permanent en particulier en mécanique des solides ( $c f .[1,4,7])$. Dans le cas des éléments finis affines, trois types de contact unilatéral sont pratiqués et donnent des résultats satisfaisants; 1'analyse 
numérique complète de leurs performances est détaillée dans [2] où un taux de convergence optimal a été exhibé pour deux des trois modèles numériques.

Parfois, il s'avère utile d'employer des élément finis d'ordre supérieur d'autant que la solution du problème de Signorini est en général plus régulière que $\mathrm{H}^{2}$ (l'espace de Sobolev standard de degré 2) au voisinage de la zone de contact. La construction de méthodes d'approximation plus précises par des éléments finis quadratiques est une voie à explorer avant d'espérer une extension aux éléments finis de type $p$ ou de type $h p$. Comme nous l'avons déjà évoqué plus haut, la difficulté principale réside dans la prise en compte des conditions de contact qui conduit souvent à des méthodes non conformes; la solution approchée ne remplit pas la condition de non-interpénétration en tous points. Dans cette Note, nous présentons les deux discrétisations par des éléments finis d'ordre deux du problème de Signorini analysées dans [3] et nous donnons les résultats de convergence optimaux démontrés moyennant des hypothèses raisonnables de régularité sur les solutions exactes. Ces deux méthodes offrent l'avantage d'être faciles à mettre en œuvre. À l'image des éléments finis affines [1,2], l'analyse de leurs performances repose sur le théorème de Falk et utilise des outils fins d'analyse fonctionnelle tels que les injections de Sobolev et de Sobolev-Morrey ainsi que des résultats d'approximation par éléments finis dans les espaces de Lebesgue $\mathrm{L}^{p}$ et dans les espaces de Hölder $\mathcal{C}^{0, \alpha}$.

\section{L'inéquation variationnelle de Signorini}

Pour alléger la présentation, nous avons choisi de décrire les différentes discrétisations et d'exposer les résultats de leur convergence pour le problème de Poisson-Signorini - qui a un intérêt propre dans le domaine de la climatisation -, étant entendu que l'extension au problème de Signorini en élasticité linéaire ne génère aucune difficulté spécifique. Soit $\Omega$ un ouvert borné de $\mathbb{R}^{2}$ à frontière lipschitzienne et $\boldsymbol{n}$ la normale extérieure à $\partial \Omega$. On suppose que le bord est partitionné en trois morceaux $\Gamma_{u}, \Gamma_{g}$ et $\Gamma_{C}$. La partie $\Gamma_{u}$, de mesure non nulle, est assujettie à des conditions de Dirichlet, le long de $\Gamma_{g}$ une condition de Neumann est prescrite à $u$ et $\Gamma_{C}$ est candidate à être en contact avec un obstacle, ce qui signifie que la zone effective de contact est contenue dans $\Gamma_{C}$. Pour toutes données $f \in \mathrm{L}^{2}(\Omega)$ et $g \in \mathrm{L}^{2}\left(\Gamma_{g}\right)$, le problème de Poisson-Signorini consiste à trouver $u$ solution du système aux dérivées partielles (1). Le cadre fonctionnel approprié pour résoudre ce problème fait intervenir le cône convexe fermé de $\mathrm{H}^{1}(\Omega)$

$$
K(\Omega)=\left\{v \in \mathrm{H}^{1}(\Omega), v_{\mid \Gamma_{u}}=0, v_{\mid \Gamma_{C}} \geqslant 0\right\} .
$$

Le formulation faible du problème de Signorini (1) conduit à l'inéquation variationnelle : chercher $u \in K(\Omega)$ tel que, pour tout $v \in K(\Omega)$,

$$
\int_{\Omega} \nabla u \nabla(v-u) \mathrm{d} \boldsymbol{x} \geqslant \int_{\Omega} f(v-u) \mathrm{d} \boldsymbol{x}+\int_{\Gamma_{g}} g(v-u) \mathrm{d} \Gamma .
$$

La forme bilinéaire et la forme linéaire figurant dans le problème faible satisfont les hypothèses du théorème de Stampacchia; il admet donc une solution unique dans $K(\Omega)$.

\section{Premier modèle d'éléments finis quadratiques}

Afin d'approcher cette inéquation variationnelle par éléments finis quadratiques on suppose que $\Omega$ est polygonal. Pour tout paramètre de discrétisation $h>0$, on considère $\mathcal{T}_{h}$, une partition de $\Omega$ en triangles (ou en quadrangles) avec une taille maximale $h$, i.e. $\bar{\Omega}=\bigcup_{\kappa \in \mathcal{T}_{h}} \bar{\kappa}$. La triangulation $\mathcal{T}_{h}$ est supposée $\mathcal{C}^{0}$-régulière au sens de [5]. Dans chaque élément $\kappa \in \mathcal{T}_{h}, \mathcal{P}_{2}(\kappa)$ désigne l'ensemble des polynômes de degré total $\leqslant 2$ (de degré $\leqslant 2$ dans chaque direction d'espace si le maillage est quadrangulaire) et $\Xi_{\kappa}$ est constitué des nœuds de Lagrange qui sont les sommets des éléments et les milieux de leurs côtés, on pose $\Xi_{h}=\bigcup_{\kappa \in \mathcal{T}_{h}} \Xi_{\kappa}$. On note $\mathcal{T}_{h}^{C}$ la «trace» de la triangulation $\mathcal{T}_{h}$ sur la zone de contact $\Gamma_{C}$ qui est 
caractérisée par la subdivision $\left(\boldsymbol{x}_{i}^{C}\right)_{0 \leqslant i \leqslant i^{*}},\left(t_{i}=\right] \boldsymbol{x}_{i}^{C}, \boldsymbol{x}_{i+1}^{C}[)_{0 \leqslant i \leqslant i^{*}-1}$ et le milieu de $t_{i}$ est $\boldsymbol{x}_{i+1 / 2}^{C}$. Une première modélisation numérique de la condition de Signorini consiste à considérer une solution discrète positive aux nœuds $\left(\boldsymbol{x}_{i}^{C}\right)_{i}$ et dont les moments sur les $\left(t_{i}\right)_{i}$ sont positifs, de sorte que le cône convexe discret $K_{h}(\Omega)$ est donné par :

$$
\begin{aligned}
K_{h}(\Omega)=\left\{v_{h} \in \mathcal{C}(\bar{\Omega}) ;\right. & \forall \kappa \in \mathcal{T}_{h}, v_{h \mid \kappa} \in \mathcal{P}_{2}(\kappa), v_{h \mid \Gamma_{u}}=0, \\
& \left.v_{h}\left(\boldsymbol{x}_{i}^{C}\right) \geqslant 0, \forall i\left(0 \leqslant i \leqslant i^{*}\right), \int_{t_{i}} v_{h} \mathrm{~d} \Gamma \geqslant 0, \forall i\left(0 \leqslant i \leqslant i^{*}-1\right)\right\} .
\end{aligned}
$$

Il est clair que $K_{h}(\Omega) \not \subset K(\Omega)$; la discrétisation est donc non conforme. Le problème aux éléments finis issu du problème continu faible (3) est l'inéquation variationnelle discrète : chercher $u_{h} \in K_{h}(\Omega)$ tel que, pour tout $v_{h} \in K_{h}(\Omega)$,

$$
\int_{\Omega} \nabla u_{h} \nabla\left(v_{h}-u_{h}\right) \mathrm{d} \boldsymbol{x} \geqslant \int_{\Omega} f\left(v_{h}-u_{h}\right) \mathrm{d} \boldsymbol{x}+\int_{\Gamma_{g}} g\left(v_{h}-u_{h}\right) \mathrm{d} \Gamma .
$$

Le théorème de Stampacchia donne l'existence et l'unicité de solution $u_{h} \in K_{h}(\Omega)$ qui dépend continûment des données $(f, g)$. L'analyse de la qualité de l'approximation est basée sur le lemme abstrait suivant :

LEMME DE FALK ([5]). - Les solutions u du problème (3) et $u_{h}$ du problème approché (4) vérifient

$$
\begin{aligned}
\left\|u-u_{h}\right\|_{\mathrm{H}^{1}(\Omega)}^{2} \leqslant C & {\left[\inf _{v_{h} \in K_{h}(\Omega)}\left(\left\|u-v_{h}\right\|_{\mathrm{H}^{1}(\Omega)}^{2}+\left\langle\frac{\partial u}{\partial \boldsymbol{n}}, v_{h}-u\right\rangle_{1 / 2, \partial \Omega}-\int_{\Gamma_{g}} g\left(v_{h}-u\right) \mathrm{d} \Gamma\right)\right.} \\
& \left.+\inf _{v \in K(\Omega)}\left(\left\langle\frac{\partial u}{\partial \boldsymbol{n}}, v-u_{h}\right\rangle_{1 / 2, \partial \Omega}-\int_{\Gamma_{g}} g\left(v-u_{h}\right) \mathrm{d} \Gamma\right)\right]^{1 / 2},
\end{aligned}
$$

$\langle\cdot, \cdot\rangle_{1 / 2, \partial \Omega}$ est le crochet de dualité entre $\mathrm{H}^{1 / 2}(\partial \Omega)$ et son dual $\mathrm{H}^{-1 / 2}(\partial \Omega)$.

Le premier terme intégral de (5) est lié à la discrétisation d'inéquations variationnelles et contribue toujours à la majoration de l'erreur globale même dans le cas d'une approximation conforme. En revanche, le deuxième terme est spécifiquement lié à la non-conformité de la méthode et disparaît pour les techniques conformes, c'est l'erreur de consistance. L'analyse de ces deux erreurs repose sur l'utilisation de l'opérateur d'interpolation $\mathcal{J}_{h}$ caractérisé par les degrés de liberté

$$
\left(v_{h}(\boldsymbol{x})\right)_{\boldsymbol{x} \in \Xi_{h} \backslash \Gamma_{C}}, \quad\left(v_{h}\left(\boldsymbol{x}_{i}^{C}\right)\right)_{0 \leqslant i \leqslant i^{*}}, \quad\left(\int_{t_{i}} v_{h} \mathrm{~d} \Gamma\right)_{0 \leqslant i \leqslant i^{*}-1} .
$$

Cet opérateur satisfait les majorations suivantes : pour tout $v \in \mathrm{H}^{\nu+1}(\Omega)$,

$$
\left\|v-\mathcal{J}_{h} v\right\|_{\mathrm{H}^{1}(\Omega)} \leqslant C h^{\nu}\|v\|_{\mathrm{H}^{\nu+1}(\Omega)}, \quad\left\|v-\mathcal{J}_{h} v\right\|_{\mathrm{H}^{1}\left(\Gamma_{C}\right)^{\prime}} \leqslant C h^{\nu+3 / 2}\|v\|_{\mathrm{H}^{\nu+1}(\Omega)} .
$$

En outre, dès que $v \in K(\Omega) \cap \mathcal{C}(\bar{\Omega})$ l'interpolé $\mathcal{J}_{h} v \in K_{h}(\Omega)$. En s'appuyant sur ces observations, l'étude menée dans [3] permet de conclure par les résultats suivants :

THÉORÈME 1. - Soit $u \in K(\Omega)$ la solution du problème de Signorini (3).

(i) On suppose que $u \in \mathrm{H}^{\nu}(\Omega)$ avec $1<\nu \leqslant 3 / 2$, alors la solution approchée $u_{h}$ est telle que

$$
\left\|u-u_{h}\right\|_{\mathrm{H}^{1}(\Omega)} \leqslant C h^{\nu-1}\left(\|u\|_{\mathrm{H}^{\nu}(\Omega)}+\|f\|_{\mathrm{L}^{2}(\Omega)}+\|g\|_{\mathrm{L}^{2}\left(\Gamma_{g}\right)}\right) .
$$


(ii) On suppose que $u \in \mathrm{H}^{\nu}(\Omega)$ avec $2<\nu \leqslant 5 / 2$, alors la solution approchée $u_{h}$ est telle que

$$
\left\|u-u_{h}\right\|_{\mathrm{H}^{1}(\Omega)} \leqslant C h^{\nu-1}\|u\|_{\mathrm{H}^{\nu}(\Omega)} .
$$

THÉORÈmE 2. - Soit $u \in K(\Omega)$ la solution du problème de Signorini (3). On suppose que l'ensemble des points de $\Gamma_{C}$ pour lesquels on passe de $u>0$ à $u=0$, soit fini.

(i) On suppose que $u \in \mathrm{H}^{\nu}(\Omega)$ avec $3 / 2<\nu<2$, alors la solution approchée $u_{h}$ est telle que

$$
\left\|u-u_{h}\right\|_{\mathrm{H}^{1}(\Omega)} \leqslant C h^{\nu-1}\|u\|_{\mathrm{H}^{\nu}(\Omega)} .
$$

(ii) On suppose que $u \in \mathrm{H}^{2}(\Omega)$, alors la solution approchée $u_{h}$ est telle que

$$
\left\|u-u_{h}\right\|_{\mathrm{H}^{1}(\Omega)} \leqslant C h|\log (h)|^{1 / 4}\|u\|_{\mathrm{H}^{2}(\Omega)} .
$$

Remarque 1. - Du point de vue de la mécanique des solides, l'hypothèse faite dans le théorème 2 , à savoir que l'ensemble des points de $\Gamma_{C}$ pour lesquels on passe de $u>0$ à $u=0$ soit fini, n'est pas contraignante; elle est pratiquement toujours remplie pour des situations qui intéressent les ingénieurs. Elle a été employée pour la première fois dans [4] pour retrouver l'optimalité dans le cas des éléments finis $\mathcal{P}_{1}$ en supposant une plus forte régularité sur $u$ au voisinage de $\Gamma_{C}\left(u \in \mathrm{W}^{1, \infty}\right)$.

\section{Un autre modèle d'éléments finis quadratiques}

Une alternative au modèle numérique précédent, et qui semble plus naturelle, est d'imposer à la solution calculée $u_{h}$ d'être positive en tous les points du maillage $\mathcal{T}_{h}^{C}$. Un tel choix conduit à considérer un cône convexe discret $\widetilde{K}_{h}(\Omega)$ différent du premier où on privilégie la condition $u_{\mid \Gamma_{C}} \geqslant 0$ au détriment de $\left.\frac{\partial u}{\partial \boldsymbol{n}}\right|_{\Gamma_{C}} \geqslant 0$ qui n'est remplie que faiblement grâce à la formulation variationnelle. Le cône convexe de travail devient alors

$$
\begin{aligned}
\widetilde{K}_{h}(\Omega)=\{ & \left\{v_{h} \in \mathcal{C}(\bar{\Omega}) ; \forall \kappa \in \mathcal{T}_{h}, v_{h \mid \kappa} \in \mathcal{P}_{2}(\kappa), v_{h \mid \Gamma_{u}}=0,\right. \\
& \left.v_{h}\left(\boldsymbol{x}_{i}^{C}\right) \geqslant 0, \forall i\left(0 \leqslant i \leqslant i^{*}\right), v_{h}\left(\boldsymbol{x}_{i+1 / 2}^{C}\right) \geqslant 0, \forall i\left(0 \leqslant i \leqslant i^{*}-1\right)\right\} .
\end{aligned}
$$

Le problème discret est analogue au problème (4), chercher $\tilde{u}_{h} \in \widetilde{K}_{h}(\Omega)$ tel que : pour tout $v_{h} \in \widetilde{K}_{h}(\Omega)$,

$$
\int_{\Omega} \nabla \tilde{u}_{h} \nabla\left(v_{h}-\tilde{u}_{h}\right) \mathrm{d} \boldsymbol{x} \geqslant \int_{\Omega} f\left(v_{h}-\tilde{u}_{h}\right) \mathrm{d} \boldsymbol{x}+\int_{\Gamma_{g}} g\left(v_{h}-\tilde{u}_{h}\right) \mathrm{d} \Gamma .
$$

La discrétisation est encore non conforme car $\widetilde{K}_{h}(\Omega) \not \subset K(\Omega)$. De plus, par la formule d'intégration de Simpson, on observe que $\widetilde{K}_{h}(\Omega) \subset K_{h}(\Omega)$; il s'ensuit que l'erreur de consistance reste inchangée. L'analyse de l'erreur d'approximation s'appuie sur l'emploi de l'opérateur d'interpolation de Lagrange et requiert davantage de technicité que pour le premier modèle numérique des conditions de Signorini. Néanmoins, les performances de cette nouvelle méthode donnent asymptotiquement les mêmes taux de convergences que les théorèmes 1 et $2(c f .[3])$ sous les mêmes hypothèses de régularités.

\section{Perspectives}

Les perspectives futures sont de deux ordres. D'abord l'extension de ce travail à la dimension trois qui semble être une tâche autrement plus difficile que pour la dimension deux.

L'étape suivante consiste à considérer des problèmes de contact unilatéral entre deux solides déformables ayant des coefficients d'élasticité différents, nécessitant l'emploi de maillages incompatibles. En combinant les outils développés dans [3] à ceux de [6], on doit pouvoir démontrer des estimations d'erreurs optimales. 


\section{Références bibliographiques}

[1] Ben Belgacem F., Numerical simulation of some variational inequalities issued from unilateral contact problems by finite element methods, SIAM J. Numer. Anal. 37 (2000) 1198-1216.

[2] Ben Belgacem F., Mixed finite element methods for Signorini's problem, (submitted).

[3] Belhachmi Z., Ben Belgacem F., Quadratic finite element approximation of the Signorini problem, (submitted).

[4] Brezzi F., Hager W., Raviart P.-A., Error estimates for the finite element solution of variational inequalities, Numer. Math. 28 (1977) 431-443.

[5] Ciarlet P.G., The Finite Element Method for Elliptic Problems, North-Holland, 1978.

[6] Hild P., Problèmes de contact unilatéral et maillages incompatibles, Thèse de l'Université Paul-Sabatier, Toulouse-3, 1998.

[7] Haslinger J., Hlaváček J., Nečas J., Numerical methods for unilateral problems in solid mechanics, in: Handbook of Numerical Analysis, Vol. IV, Part 2, Ciarlet P.G., Lions J.-L. (Eds.), North-Holland, 1996. 